\title{
Clinical Trials Regulations in India
}

\section{Hitt Sharma ${ }^{1 *}$ and Sameer Parekh²}

${ }^{1}$ Additional Director, Department of Medical Affairs and Clinical Trials, Serum Institute of India Ltd., Pune, India ${ }^{2}$ Manager, Clinical Trials, Serum Institute of India Ltd., Pune, India

\section{Editorial}

India is a preferred destination for most of the leading global companies for carrying out clinical trials. Apart from specific elements of the Indian market that attract global players, the main aspect that puts the country at an advantage is the notable momentum adopted by the Indian Council of Medical Research (ICMR) and the Central Drugs Standard Control Organization (CDSCO) - Directorate General of Health Services, Government of India, in tandem with global regulatory guidelines - in building up a robust regulatory system, considering India's involvement in global GCP has only happened in the past decade. Most of the concerns were looked into detail and new guidelines and laws were introduced to strengthen the existing regulations. Thus, a balance between the ethics and trade has been created for both the government and the private sector.

Due to India's advantage of strategic location, skilled manpower, low cost, good medical infrastructure and English speaking population, the number of clinical trials were augmented from 1300 in 2009 to more than 1900 by 2013 . The regulatory bodies therefore recognized the need to frame guidelines and regulatory approval processes on a par with international standards.

Many of the new laws, guidance documents, notifications and initiatives for regulating pharmaceutical industry were in the charts for quite a long time. Now, Indian regulatory authorities have started looking into speedy implementation and providing support in terms of necessary infrastructure and investment. Also, setting up of an appropriate administrative and monitory mechanism is on the cards to ensure its functioning.

\section{Regulatory Bodies, Framework and Procedures}

In India, the Central Drugs Standard Control Organization (CDSCO) which comes under the Ministry of Health and Family Welfare is the main body which works on development of regulatory procedures and standards for drugs, cosmetics, diagnostics and devices. It lays down regulatory guidance by amending acts and rules; and regulates new drug approval process. Its main objective is to standardize clinical research and bring safer drugs to the Indian market.

The Drug Controller General of India DCG(I) is responsible for giving regulatory permissions for the conduct of clinical trials and is responsible for approval of marketing licenses for drugs in India. Alongwith the DCG(I) office, there are other governmental bodies involved in the pharmaceutical regulations of the new drugs (Figure 1). When an application is filed, the office of the DCG(I) reviews it; the required time for approval depends on the trial's regulatory status in other countries. To expedite the approval process, for studies already approved by other countries' regulatory agencies, the DCG(I) office has divided all applications into two categories: A and B. Category A includes clinical trials whose protocols have been approved by EMEA or regulatory agencies in the US, UK, Switzerland Australia, Canada, Germany, South Africa or Japan. For such studies, permission is granted, accepting the protocol approval of those countries. For category A applications, review and approval are projected to take two to four weeks. The category B clinical trial applications, are reviewed following the regular approval system by an expert committee. This process usually takes eight-twelve weeks for approval of application. This review time does not include potential delays due to incomplete applications or the time taken by sponsor's response to queries raised by the regulatory authority. Along with the application, summarized information in the form of an Investigators' Brochure comprising detailed pharmacology, toxicology and clinical experience data, if any, is submitted. The DCG(I) at times also requests guidance from other independent government agencies viz., ICMR or Department of Biotechnology (for biotech products) on a case-by-case basis, thus extending the review period.

DCG(I) approval is also required to receive a test license to import trial supplies, which takes 2 weeks. All the above process in parallel requires a total of 14 weeks. After clinical trial approval is granted by the DCG(I), additional 2-4 weeks are required for the approval of director general of foreign trade (DGFT), to export of blood samples. However, these timelines again depend upon the documentation submitted and may delay the process until the corrections/ concerns raised by regulatory authority are addressed and received.

Alongwith the application submitted to the DCG(I) office, parallel submission can be made to the ethics committees of would-be sites, which are usually hospitals and/or clinics. Ethics committees, generally which follow the ICH-GCP guidelines and schedule Y of the Drugs and Cosmetic Act of 1945. These Ethics committees are usually affiliated with study sites; or sometimes they function as independent ethics

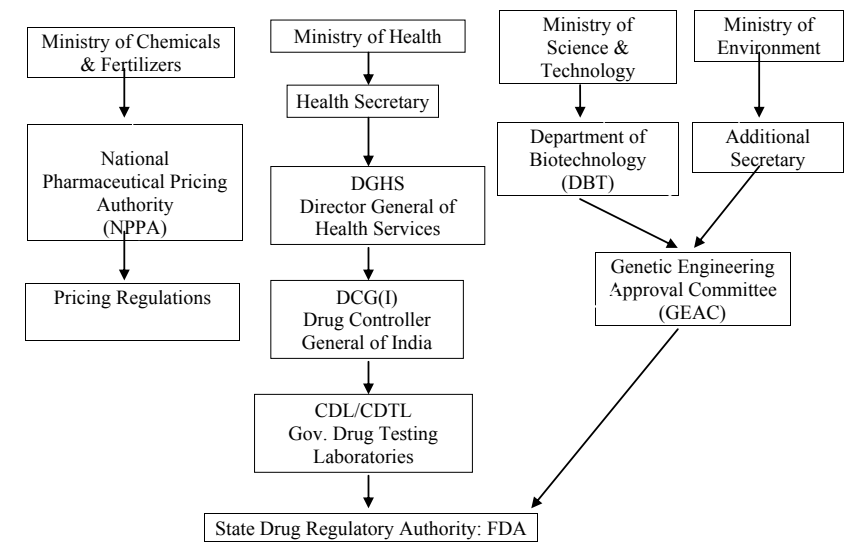

Figure 1: Regulatory bodies in India involved in pharmaceutical regulations.

*Corresponding author: Hitt Sharma, Additional Director, Department of Medical Affairs and Clinical Trials, Serum Institute of India Ltd., Pune- 411028 Maharashtra, India, Tel: +91- 20- 26602451; Fax: +91- 20- 26998146; E-mail drhjs@seruminstitute.com, hittsharma@gmail.com

Received October 31, 2012; Accepted November 01 2012; Published November 03, 2012

Citation: Sharma H, Parekh S (2012) Clinical Trials Regulations in India Pharmaceut Reg Affairs 1:e118. doi:10.4172/2167-7689.1000e118

Copyright: $\odot 2012$ Sharma $\mathrm{H}$, et al. This is an open-access article distributed under the terms of the Creative Commons Attribution License, which permits unrestricted use, distribution, and reproduction in any medium, provided the original author and source are credited. 
committees. The standard time required for ethics committee approval is 4 to 6 weeks. Many study sites have scientific review committees (SRC) to review the scientific rationale of the study. Once the study is passed through and approved by the SRC then it is submitted to the ethics committee for their assessment.

The key regulations under which the Ministry of Health and family Welfare, Directorate General of Health Services functions is as given in table 1.

\section{Priorities of CDSCO}

India's business and regulatory climate has undergone a dramatic change in the past 2-3 years. Regulations are updated to harmonize with international standards, esp. US FDA-like regulatory body. In years to come, CDSCO has made it clear about it's priorities to enhance the current mechanism.

- Establish single window clearance for approvals.

- Fix timelines for each application (2-6 Weeks).

- New drug application status on the web -update fortnightly.

- Subject experts-reviewers -internal / external.

- Staff \& infrastructure at one site.

- Continuous training for the officers.

\section{Clinical Trial Regulation}

Status of the new drug would define the procedure for applying for marketing approval. It can be largely classified into following categories: a) new drug substances discovered that are already approved/marketed in other countries; b) new drug substances discovered that are not approved/marketed in other countries; and c) new drug substances discovered in India.

In case of the A category, it is sufficient if Phase III (therapeutic confirmatory) trials are conducted in a large number of patients with multiple site and compared with a standard drug, to confirm efficacy and safety. For the category B, permission for clinical trials is given with a "phase lag". For example, phase I study for a new drug is allowed only if the drug has completed phase I in other country and moved to the next phase. In the case of new drug substances discovered in India i.e. category C, phase I clinical trials need to be carried out in India. The phase I trials are carried out on healthy, adult human volunteers to assess the safety profile and adverse drug reactions, etc.

However, this has been recently revised, and now in the case of new drug substances not yet approved or marketed in other countries, Schedule Y now allows clinical trials to be carried out in India, concurrently with the trials abroad.

In the last decade, due to illiteracy and lack of awareness, it was possible that the volunteers were not adequately informed about the risk they are undertaking as well as their rights.

Some feared that volunteers are forced by poverty to offer themselves as 'guinea pigs' and take part in trial. However, these lacunas coupled with clinical trial process are now history and now India has appropriate provisions to ensure that human subjects used for the trials are informed well and their participation is voluntary. Compliance to International Conference on Harmonization-Good Clinical Practice (ICH-GCP) norms, well qualified and trained investigators, a growing population of experienced clinical research monitors and exposure to international systems has enabled the Indian industry to become an alternative to the costly sites across the world. The pace of drug trials in the country has forced a non-profit organization in US viz., Clinical Data Interchange Standards Consortium, to look at setting up a division here.

\section{Recent Initiatives and Priories by CDSCO for Ethical and Quality Clinical Research}

Since 2008-09 CDSCO has taken up help from number of World Health Organization (WHO), USA FDA, Health Canada, ANVISA Brazil and South Africa to develop methodical approach for clinical trial regulatory systems. Since then many changes, amendments were drafted and finalized and a road map was developed to strength the regulatory process.

- Implementation of CTD format for biological \& introduction of CTD format for new chemical entities.

- Building of regulatory framework to allow phase 0 (micro dosing) and phase- 1 studies in the country in a phase wise manner.

- Mandatory registration of clinical trial on clinical trial registry since June 2009.

- 12 New Drug Advisory Committees (NDAC) were constituted to examine the applications for permissions for clinical trials and approvals for new drugs.

- Draft notification for registration of clinical research organizations issued on Jan 2011.

- Draft guidelines for reporting Serious Adverse Event- May 2011.

- Guidance on Clinical Trial Inspection-November 2010.

- Registration of Ethics Committee will be mandatory.

- Drafter guidelines for enhancement of the responsibilities of Ethics Committee, Investigator and Sponsor to ensure that financial compensation as well as medical care is provided to the trial subjects who suffer trial related injury or deaths.

- Schedule M III guidelines for devices have been posted on website which are inline with GHTF.

- Rules to be amended and medical devices would be separated

Ministry of Health \& Family Welfare Directorate General of Health Services

Regulates import, manufacture, distribution and sale Cosmetics Act, 1940 of drugs and cosmetics whether imported or made in India DPCO, 1995 is an order issued by the Gol under Section 3 of the Essential Commodities Act, 1955 to regulate the prices of drugs

The Drugs Price Contro Order (DPCO), 1995 Currently 74 bulk drugs and their corresponding formulations are under price control

The Drugs and Magic Remedies (Objectionable Prohibits the advertising of remedies alleged to possess magic qualities

Aeals with levy and collection of taxes on medicinal and toilet preparations containing alcohol, opium, Indian hemp, or any other narcotic drug

The Pharmacy Act,

Regulates the profession of pharmacy and deals 1948 with various pharmacy issues such as professional education and requirements for registration

Patents (Amendment) Recognition of product patents for food, drug, and Rule 2005 pharmaceuticals from January 1, 2005

Table 1: Key regulations. 
definition of drug. Provision for overseas inspections under Rule24-A(5). The overseas inspections from 2011 were regularly conducted in different countries from where imports of drugs are taking place.

- Good Laboratories Practices were notified under notification GSR 780(E) dated 10.11.2008 and became operative since 1.11.2010.

\section{Conclusion}

From now onwards, India is poised to lead from the front in the global clinical development process. Its regulatory process has become more streamlined. After the success of information technology and service industries like BPO, India has now developed a strong reputation and meets professional global parameters, in clinical research. The Indian regulatory agencies are working closely with international bodies to update the clinical trial process and the regulatory system. With the support and experience of international regulatory agencies, the Indian regulatory system has now become robust, efficient and effective. The fact that English is one of the primary languages gives India an advantage over China, other countries in the region, and Eastern Europe. 